\title{
Magnetoelastic Sensors - Status, Commercial Readiness, and Outlook
}

\author{
Jens Müller, NCTE AG, Inselkammerstrasse 4, D-82008 Unterhaching, Germany \\ jens.mueller@ncte.de
}

\begin{abstract}
Torque / load sensors will play an increasingly important role in many applications, provided that functional requirements and cost targets for serial production are met. They enable critical benefits such as efficiency improvements, weight savings, operational safety, enhanced productivity, and cost savings. Magneto-elastic sensors, being truly non-contact based, have inherent advantages over traditional solutions based on strain gauges regarding robustness and cost structures. Recently, they have emerged as a viable, commercially proven alternative in a number of industry sectors with demanding requirements. The technical and commercial readiness level of magneto-elastic sensor technology is presented and discussed in this presentation. Also, an outlook is presented regarding recent developments in making magneto-elastic sensor technology applicable to non-magnetic metals.
\end{abstract}

Key words: torque sensor, mechanical force, shear, magnetoelastic, strain gauge

\section{Introduction}

Force sensors (torque, shear force, load) are key components in many industries like e.g. automotive or aviation. Initially, they were primarily used in research \& development, i.e. for testing and evaluation purposes rather than in serial products "on-board". The significance of force sensors has been constantly increasing because of more stringent requirements re. weight savings, efficiency gains, safety maximization, cost savings across a variety of industries.

\section{Historical Background}

Traditionally, strain-gauge systems have been the preferred approach for measuring mechanical forces along moving components. Strain gauges represent without any doubt a well-developed, advanced solution for accurate measurements, and a mature landscape of established industry players has evolved around this technology. However, several reasons have prohibited strain gauge systems from entering volume markets of serial OEM products.

From a technological perspective, moving components present challenges for power supply and signal transmission to and from the strain gauge. As wiring is typically not an option, either slip rings or telemetry solutions must be employed. Slip rings have lifetime limitations especially under harsh operating conditions or in challenging environments; telemetry solutions are mostly incompatible with the cost targets of an OEM component in volume production. Also, mechanical fixation of strain-gauges tends to have durability limitations in demanding environments, e.g. when exposed to extreme temperature cycling, shock, or being submerged in aggressive fluids. As a result, strain-gauge systems are widely used in protected laboratory environments and test equipment, including test vehicles and certain price-insensitive niche applications, but their usage "on-board" serial products is extremely limited.

\section{Magneto-elastic Sensors: Concept and Advantages}

Against this background, magneto-elastic sensors have evolved as a viable alternative to strain-gauge systems, especially with regard to employment in volume production of more price-sensitive equipment. The underlying magneto-elastic effect, or "magnetostriction", was discovered close to 200 years ago; only recently have they entered the marketplace. The compelling advantage from a technology perspective is that magneto-elastic sensors feature cost structures that make them compatible with mass production. An existing component, e.g. a shaft, is converted to the primary force sensor only by magnetic encoding. This process step does not add any material cost, but merely represents a processing step which can be done within seconds. As a result, there is typically no extra material cost associated with the primary sensor. The secondary sensor consists of a 
number of magnetic field detectors and a small printed circuit board for signal processing. This short description explains why the cost structures of magneto-elastic force sensors are attractive for OEM use: overall system complexity, part count, and number of handling steps are extremely low. This sensor principle is easy to integrate into demanding OEM products. An existing component (which is required anyhow for the mechanical function) is converted into the primary sensor within seconds, avoiding the costly application of strain gauges and associated telemetry circuitry. These attractive cost structures make magneto-elastic force sensors ideal for volume applications, such as torque sensors for the drivetrain of pedelecs ("E-bikes"). In this segment alone, over 1 million magneto-elastic torque sensors have been sold into a highly price-sensitive end-user market. Such volume markets are inaccessible by strain-gauge systems.

\section{Magneto-elastic Sensors: Limitations and Outlook}

The underlying working principle of magnetoelastic sensors requires that a permanent magnetic encoding be present on the surface of the component to be measured. The magnetic field strengths employed are typically on the order of $1 \mathrm{mT}$, which is roughly comparable to the earth's magnetic field. Since the magnetization needs to last for several decades, the selection of suitable metals is somewhat limited. Many steel alloys commonly used for mechanical parts of e.g. gearboxes or driveshafts fulfill these requirements. Nonferromagnetic materials today represent a hurdle towards the use of magneto-elastic sensing - which recently has been overcome successfully by application of a suitable coating on the surface. By this approach, it is now possible to use non-ferromagnetic materials (e.g. titanium) as a substrate to be converted into a magneto-elastic force sensor.

\section{Conclusion}

Magneto-elastic sensors have emerged as a commercially viable alternative to strain-gauge systems for measuring torque, shear, and other loads. Their very low system complexity, the ease of integration into OEM products, and inherently low cost structures make them ideal for volume production. With over 1 million such sensors in actual use in end-user environments, they have successfully entered the market and proven technical and commercial readiness. Magneto-elastic sensors have enabled sophisticated force measurement to now move down the value chain in many large industries - from R\&D and testing applications into volume and mass production. 